\title{
Reducing Codebook Loss with Reconfigurable Receive Antennas
}

\author{
Uzma Afsheen ${ }^{\dagger}$, Peter J. Smith ${ }^{\circ}$ and Philippa A. Martin ${ }^{\dagger}$, \\ $\dagger$ Department of Electrical and Computer Engineering, University of Canterbury, \\ Private Bag 4800, Christchurch 8140, New Zealand. \\ philippa.martin@canterbury.ac.nz, uzma.afsheen@pg.canterbury.ac.nz. \\ - School of Mathematics and Statistics, Victoria University of Wellington, \\ PO Box 600, Wellington 6140, New Zealand. peter.smith@vuw.ac.nz.
}

\begin{abstract}
In this paper, a reconfigurable antenna codebook feedback scheme is investigated to reduce precoding codebook loss without increasing the number of feedback bits. Analysis and simulations show that increasing the number of receive antenna states $(\boldsymbol{S})$ has the same effect as codebook size expansion when the codeword and state selection is based on minimizing the distance between channel and codeword. In addition, we propose an optimum selection approach. The SNR and rates achieved with perfect feedback in a traditional non-reconfigurable antenna system, can be exceeded with only $\boldsymbol{S}=\mathbf{2}$, using the proposed optimum selection. In fact doubling $\boldsymbol{S}$ gives an approximate SNR gain of $\mathbf{4 0} \%$ compared to $S=1$. We also consider the effects of channel estimation errors and correlated antenna states. We show that the performance losses due to correlation can be quantified with a simple closed form result.
\end{abstract}

\section{Index Terms}

Reconfigurable antenna, limited feedback, precoding, codebook, MISO, beamforming. 


\section{INTRODUCTION}

In this paper we consider a limited feedback (FB) multi-input single-output (MISO) precoding system equipped with reconfigurable antennas. Reconfigurable antennas [1], [2], [3] have great potential in small hand held devices and are very attractive due to their ability to change radiation pattern, frequency of operation or polarization. This allows the propagation channel to be altered dynamically and deliberately, so that different channel conditions can be experienced. Many forms of reconfigurable antenna have been proposed in the literature. An adaptive resolution array was described in [4] to convert the actual scattered channel matrix into a channel which exhibits optimum capacity scaling properties. Polarization states were considered in [5] where the state switching is performed by microelectromechanical actuators. Pattern diversity in an antenna array is investigated in [17] where varying the loads on parasitic elements in the array creates different channels. Pattern diversity is also explored in [7] where PIN diode switches reconfigure the antenna length, hence altering the coupling and radiation patterns. Limits of reconfigurable antennas are studied in [8] where the performance of a finite volume array is investigated as the number of states and the number of elements both grow. In this scenario, a region of diminishing returns is demonstrated. In this paper, we take a general approach to the problem and model the different channels delivered by the reconfigurable arrays rather than any one specific mechanism.

A significant amount of research has recently been focused on limited feedback precoding systems [9], [10]. Codebook transmit beamforming is now a part of LTE standards [11]. The codebook size, $2^{B}$, defined by the number of feedback bits $(B)$, impacts performance and is related to system diversity [12]. The trade-off between performance and the amount of feedback has been extensively researched [9], [10].

In terms of limited feedback performance analysis, there is now a considerable body of work, mainly for single user systems. Results are available for independent and identically distributed (i.i.d.) Rayleigh channels [13], [14], [15], [16], [17], correlated Rayleigh channels [18] and double-sided correlated Rayleigh channel matrices [19]. These results apply to MISO channels [15], [16], [18] and multiple-input multiple-output channels [13], [14], [17], [19]. The analytical

approaches are based on random vector quantization (RVQ) [13], [15], [17], [19] approaches or on distortion bounds and approximations [14], [16], [18]. Particular contributions include bounds 
on the ergodic capacity loss due to limited feedback [13], bounds and approximations on outage probability and the mean SNR loss due to limited feedback [14], bounds on capacity loss due to spatial correlation [18], asymptotic (large system) results on beamforming capacity [17] and bounds on SNR loss and mutual information loss [19]. For the case of i.i.d. Rayleigh MISO systems, exact results were obtained in [15] covering mean SNR, outage probability, ergodic capacity and average bit error probability.

We propose a limited feedback precoding system employing reconfigurable receive antennas. We aim to achieve the performance of a given value of $B$ while using fewer feedback bits. We consider both perfect and imperfect channel state information (CSI) at the receiver. The receiver compares the normalized reconfigurable channel with the entries in a codebook and feeds back just the index of the nearest codebook entry. With limited codebook cardinality, it is virtually impossible to find a codebook entry at zero distance from the corresponding channel values and a loss is incurred [20]. Increasing the number of possible channel conditions instead of increasing the codebook size and required feedback bits, is the basis of our underlying approach towards minimizing the loss due to codebook mismatch. This is particularly suitable for systems with fixed feedback bits, but variable training length. Overall, the training length can be reduced at the cost of imperfect CSI, but we can still achieve gains from reconfiguration. We will consider the effect of channel estimation errors.

Increasing the number of possible channel conditions while having limited codebook cardinality, gives new degrees of freedom which can be used to reduce codebook/quantization loss and give an overall improvement in SNR due to increased channel power if the channel states are carefully selected. The optimum approach (OP) proposed here maximizes both the channel power and the direction by carefully selecting the channel state and the codebook codeword. We also consider two more selection approaches called channel direction (CD) and channel power $(\mathrm{CP})$, to investigate the source of the gains achieved by the optimum method. Finally, we investigate the impact of correlated states on performance. We show that a simple closed form result can be used to measure the number of equivalent independent states in the correlated case. The performance of a system with this reduced, equivalent, number of independent states is shown to be close to the performance of the full system with correlated states.

The paper is organized as follows. Section II introduces the reconfigurable codebook based MISO system and describes three codeword and state selection techniques. Section III gives the 
analytical forms of the expected SNRs for the selection approaches and shows that reconfiguration is equivalent to a bigger codebook size. Section IV considers correlated antenna states and simulation results follow in Section V for perfect and imperfect CSI. Section VI presents conclusions.

\section{System Model}

We consider a single user MISO feedback system employing single stream transmission using fixed LTE [11], Grassmannian [12] and RVQ codebooks [21]. The receiver and transmitter share a common codebook and an error free, zero delay feedback link is assumed.

\section{A. Reconfigurable codebook based MISO System Model}

Let there be $N_{t}$ transmit antennas in an $N_{t} \times 1$ MISO system, where the receive antenna is equipped with a reconfigurable antenna that has $S$ uncorrelated channel propagation states. This results in $S$ possible channel vectors. The channel vector corresponding to the $s^{\text {th }}$ state is defined as

$$
\mathbf{h}_{s}=\left[\begin{array}{llll}
h_{1}^{s} & h_{2}^{s} & \cdots & h_{N_{t}}^{s}
\end{array}\right],
$$

where $h_{k}^{s}$ denotes the channel coefficient between the $k^{\text {th }}$ transmit antenna and the receive antenna. The overall set of possible channel vectors can be given as $\left\{\mathbf{h}_{1}, \mathbf{h}_{2}, \ldots, \mathbf{h}_{S}\right\}$, where each $\mathbf{h}_{s}$ is assumed independent. The case of correlated channel vectors is considered in Section IV. For each transmission, the state, $s$, and hence $\mathbf{h}_{s}$ is chosen based on the selection techniques discussed later. We assume a frequency flat quasi-static Rayleigh fading channel, where each $h_{k}^{s} \sim \mathcal{C N}(0,1)$. First we assume perfect CSI at the receiver, where $\mathbf{h}_{s}=\sqrt{\lambda_{s}} \mathbf{v}_{s}^{\dagger}, \lambda_{s}=\sum_{k=1}^{N_{t}}\left|h_{k}^{s}\right|^{2}$ is the instantaneous channel power, $\mathbf{v}_{s}^{\dagger}=\left[\begin{array}{llll}h_{1}^{s} & h_{2}^{s} & \cdots & h_{N_{t}}^{s}\end{array}\right] / \sqrt{\sum_{k=1}^{N_{t}}\left|h_{k}^{s}\right|^{2}}$ is a row vector representing the normalized channel direction and ${ }^{\dagger}$ denotes the Hermitian transpose. The transmitter encodes the data, $z$, by a precoding vector, $\mathbf{w}_{p}$, which is selected from a codebook, given by $\mathbb{C}=\left\{\mathbf{w}_{1}, \mathbf{w}_{2}, \ldots, \mathbf{w}_{2^{B}}\right\}$, such that $\mathbf{w}_{p}^{\dagger} \mathbf{w}_{p}=1$ and each codeword is a $N_{t} \times 1$ vector. The system input output relationship can be modelled as

$$
r=\mathbf{h}_{s} \mathbf{w}_{p} z+n,
$$

where $r$ is the received scalar signal, $\mathbf{w}_{p} z$ is the $N_{t} \times 1$ transmitted signal and $n$ is an i.i.d. $\mathcal{C N}\left(0, \sigma^{2}\right)$ noise term. The codeword index, $p$, identifying the desired beamforming vector is 
conveyed from the receiver to the transmitter using $B$ feedback bits. We assume a total transmit power, $P_{t}=\mathbf{E}\left[|z|^{2}\right]$, and the noise power is $\mathbf{E}\left[|n|^{2}\right]=\sigma^{2}$, where $\mathbf{E}[$.$] denotes expectation. Also,$ we define the transmit signal to noise ratio, $\gamma=\mathbf{E}\left[|z|^{2}\right] / \sigma^{2}$. Then, the received signal to noise ratio $(\mathrm{SNR})$ is given by

$$
\operatorname{SNR}_{p, s}=\lambda_{s}\left|\mathbf{v}_{s}^{\dagger} \mathbf{w}_{p}\right|^{2} \gamma
$$

In (3), each state of the receive antenna, $s$, gives a different channel power, $X_{s}=\lambda_{s}$, and we see there is no ceiling on performance as $\mathbf{S N R}_{p, s}$ increases with $\gamma$. However, there is an SNR loss factor due to codebook mismatch given as $Y_{p, s}=\left|\mathbf{v}_{s}^{\dagger} \mathbf{w}_{p}\right|^{2}$. Then, (3) can be written as

$$
\operatorname{SNR}_{p, s}=X_{s} Y_{p, s} \gamma
$$

For the perfect feedback (PFB) case, the loss factor $Y_{p, s}=1$, whereas for a codebook feedback system $Y_{p, s}<1$. The system rate is given as

$$
\mathcal{R}=\log _{2}\left(1+\mathrm{SNR}_{p, s}\right) .
$$

The imperfect CSI is modelled as [22]

$$
\hat{h}_{k}^{s}=\eta h_{k}^{s}+\sqrt{1-\eta^{2}} \epsilon \text {, }
$$

where $\hat{h}_{k}^{s}$ is imperfect CSI, $\epsilon$ is an independent zero mean complex Gaussian variable with variance 0.5 per dimension and $\eta$ is the correlation between $\hat{h}_{k}^{s}$ and $h_{k}^{s}$.

\section{B. Codeword and State Selection Methods}

1) Maximizing Channel Direction (CD): This approach uses the channel with the best direction. In this approach, the direction is maximized over all states and codewords $\mathbf{w}_{p}$. The index of the best codeword, $p^{*}$, is fed back to the transmitter, and the receiver is set to state $s^{*}$, where

$$
\left(p^{*}, s^{*}\right)=\underset{\substack{p \in\left\{1,2, \ldots, 2^{B}\right\} \\ s \in\{1,2, ., S\}}}{\arg \max }\left\{\left|\mathbf{v}_{s}^{\dagger} \mathbf{w}_{p}\right|^{2}\right\}
$$


2) Maximizing Channel Power (CP): This approach maximizes the channel power resulting in channel $\mathbf{h}_{s^{*}}$ being used for transmission, where

$$
s^{*}=\underset{s \in\{1,2, . ., S\}}{\arg \max }\left\{\left\|\mathbf{h}_{s}\right\|^{2}\right\} .
$$

Given $s^{*}$, the best codeword, $\mathbf{w}_{p^{*}}$, is selected based on the best SNR such that

$$
p^{*}=\underset{p \in\left\{1,2, . ., 2^{B}\right\}}{\arg \max }\left\{\mathrm{SNR}_{p, s^{*}}\right\}=\underset{p \in\left\{1,2, . ., 2^{B}\right\}}{\arg \max }\left\{X_{s^{*}}\left|\mathbf{v}_{s^{*}}^{\dagger} \mathbf{w}_{p}\right|^{2}\right\} .
$$

This approach maximizes channel power over all states and then uses the best codeword index for that state.

3) Optimum Approach (OP): In the optimum approach, the codeword, $\mathbf{w}_{p}$, and the state are selected such that the codeword index $p=p^{*}$ and CPS $s=s^{*}$ satisfy

$$
\left(p^{*}, s^{*}\right)=\underset{\substack{p \in\left\{1,2, \ldots, 2^{B}\right\} \\ s \in\{1,2, . ., S\}}}{\arg \max }\left\{\mathrm{SNR}_{p, s}\right\}
$$

This approach simultaneously maximizes both $X_{s}$ and $Y_{p, s}$ over all codewords and states.

\section{ANALYSIS}

In order to understand the different selection methods, and quantify the gains obtained from channel direction and channel power, we now derive the expectation of the received SNR. To make analytical progress, RVQ codebooks are assumed here.

\section{A. Maximizing Channel Direction}

When maximizing $\mathrm{SNR}_{p, s}$ for the channel direction we maximize only over the loss factor term $Y_{p, s}$ and $X_{s}$ is ignored in (4). Since $X_{s}$ and $Y_{p, s}$ are independent, we can write

$$
\operatorname{SNR}_{C D}=X \max \left\{Y_{p, s}\right\} \gamma
$$

where $X$ is an arbitrary channel power and

$$
\mathbf{E}\left[\operatorname{SNR}_{C D}\right]=\mathbf{E}[X] \mathbf{E}\left[\max \left\{Y_{p, s}\right\}\right] \gamma
$$


Assuming RVQ codebooks of size $2^{B}$, the CDF, PDF and expectation of an arbitrary $Y$ are given by [21],

$$
\begin{aligned}
F_{Y}(y) & =\left(1-(1-y)^{N_{t}-1}\right)^{2^{B}}, \\
f_{Y}(y) & =2^{B}\left(1-(1-y)^{N_{t}-1}\right)^{2^{B}-1} \\
& \times\left(N_{t}-1\right)(1-y)^{N_{t}-2}, \\
\mathbf{E}[Y] & =1-2^{B} \beta\left(2^{B}, \frac{N_{t}}{N_{t}-1}\right),
\end{aligned}
$$

where $\beta(.,$.$) is the Beta function. Then, using (13)$, it is straightforward to show that the CDF of $\max \left\{Y_{p, s}\right\}$ for $s=1,2, . ., S$ is equal to

$$
\left(F_{Y}(y)\right)^{S}=\left(1-(1-y)^{N_{t}-1}\right)^{2^{B} S} .
$$

Note that the CDF in (16) is the same as that for a non-reconfigurable antenna codebook feedback system with a codebook size of $2^{B} S$. From (15) it follows that

$$
\mathbf{E}\left[\max \left\{Y_{p, s}\right\}\right]=\left(1-2^{B} S \beta\left(2^{B} S, \frac{N_{t}}{N_{t}-1}\right)\right) .
$$

Also, $X=\sum_{k=1}^{N_{t}}\left|h_{k}\right|^{2}$ has a $\chi^{2}$ distribution, assuming an i.i.d. Rayleigh fading channel, so that $\mathbf{E}[X]=N_{t}$ and

$$
\mathbf{E}\left[\mathrm{SNR}_{C D}\right]=N_{t}\left(1-2^{B} S \beta\left(2^{B} S, \frac{N_{t}}{N_{t}-1}\right)\right) \gamma
$$

Note that as $X$ is an arbitrary channel power, the superscript in $h_{k}^{s}$ has been dropped.

\section{B. Maximizing Channel Power}

In the case of maximizing the channel power, we maximize only over $X_{s}$ and $Y_{p, s}$ is ignored. Since $\mathrm{X}$ and $\mathrm{Y}$ are independent we have

$$
\mathrm{SNR}_{C P}=\max \left\{X_{s}\right\} Y \gamma
$$

where $Y$ is an arbitrary SNR loss factor,

$$
\mathbf{E}\left[\mathrm{SNR}_{C P}\right]=\mathbf{E}\left[\max \left\{X_{s}\right\}\right] \mathbf{E}[Y] \gamma,
$$


and $\mathbf{E}[Y]$ is given by (15).

The CDF of $\max \left\{X_{s}\right\}$ is the CDF of the maximum of $S \chi^{2}$ variables having PDF equal to

$$
f_{X}(x)=\frac{x^{N_{t}-1} e^{-x}}{\left(N_{t}-1\right) !}
$$

and $\mathrm{CDF}$ equal to

$$
F_{X}(x)=1-\sum_{m=0}^{N t-1} \frac{x^{m}}{m !} e^{-x}=1-T
$$

The CDF of $\max \left\{X_{s}\right\}$ with $s=1,2, \ldots S$, is $\left(F_{X}(x)\right)^{S}$ and the PDF is $S F_{X}(x)^{S-1} f_{X}(x)$. Hence, using the above equations,

$$
\mathbf{E}\left[\max \left\{X_{s}\right\}\right]=\int_{0}^{\infty} x S\left(F_{X}(x)\right)^{S-1} f_{X}(x) d x
$$

Also, from (22) we can write

$$
\begin{aligned}
\left(F_{X}(x)\right)^{S-1} & =(1-T)^{S-1}=\sum_{\alpha=0}^{S-1}\left(\begin{array}{c}
S-1 \\
\alpha
\end{array}\right)(-1)^{\alpha} T^{\alpha}, \\
T^{\alpha} & =\sum_{m_{1}=0}^{N_{t}-1} \cdots \sum_{m_{\alpha}=0}^{N_{t}-1} \frac{x^{\sum_{g=1}^{\alpha}\left(m_{g}\right)} e^{-\alpha x}}{\prod_{g=1}^{\alpha}\left(m_{g} !\right)} .
\end{aligned}
$$

Substituting (21), (24) and (25) in (23) gives

$$
\begin{aligned}
\mathbf{E}\left[\max \left\{X_{s}\right\}\right] & =S \int_{0}^{\infty} \frac{x^{N_{t}} e^{-x}}{\left(N_{t}-1\right) !} \sum_{\alpha=0}^{S-1}\left(\begin{array}{c}
S-1 \\
\alpha
\end{array}\right) \\
& (-1)^{\alpha} \sum_{m_{1}} \ldots \sum_{m_{\alpha}} \frac{x^{\sum_{g=1}^{\alpha} m_{g}} e^{-\alpha x}}{\prod_{g=1}^{\alpha} m_{g} !} d x \\
& =\frac{S}{\left(N_{t}-1\right) !}\left[N_{t} !+\sum_{\alpha=1}^{S-1}\left(\begin{array}{c}
S-1 \\
\alpha
\end{array}\right)(-1)^{\alpha} \sum_{m_{1}=0}^{N_{t}-1} \cdot .\right. \\
& \left.. \cdot \sum_{m_{\alpha}=0}^{N_{t}-1} \frac{\left(N_{t}+\sum_{g=1}^{\alpha} m_{g}\right) !}{\prod_{g=1}^{\alpha}\left(m_{g} !\right)(\alpha+1)^{N_{t}+\sum_{g=1}^{\alpha} m_{g}+1}}\right] .
\end{aligned}
$$


Hence,

$$
\begin{aligned}
\mathbf{E}\left[\mathrm{SNR}_{C P}\right] & =\left(1-2^{B} \beta\left(2^{B}, \frac{N_{t}}{N_{t}-1}\right)\right) \frac{S}{\left(N_{t}-1\right) !} \\
& {\left[\begin{array}{c}
N_{t} !+\sum_{\alpha=1}^{S-1}\left(\begin{array}{c}
S-1 \\
\alpha
\end{array}\right)(-1)^{\alpha} \sum_{m_{1}=0}^{N_{t}-1} \cdots \sum_{m_{\alpha}=0}^{N_{t}-1} \\
\\
\\
\prod_{g=1}^{\alpha}\left(m_{g} !\right)(\alpha+1)^{N_{t}+\sum_{g=1}^{\alpha} m_{g}+1}
\end{array}\right] \gamma . }
\end{aligned}
$$

\section{Correlated Channel Propagation States}

In this Section we consider the effects of correlated states. For example, in [5] the authors propose a correlation of 0.3 between the two polarization states. In the correlated scenario, the set of possible channel vectors is $\left\{\mathbf{h}_{1}, \mathbf{h}_{2}, \ldots, \mathbf{h}_{S}\right\}$, where the elements of each $\mathbf{h}_{s}$ are i.i.d. $\mathcal{C N}(0,1)$, but the elements of $\mathbf{h}_{s}$ and $\mathbf{h}_{t}$ are not independent for $s \neq t$. We consider the two correlation structures: exponential correlation (EXC) and equal correlation (EQC). With the exponential model [24], the state number has a physical interpretation such that $\mathbf{h}_{1}$ is more strongly correlated with $\mathbf{h}_{2}$ than with $\mathbf{h}_{3}$. Hence the labelling of the channels relates to how similar the channels are. Here, $\mathbf{E}\left[\left(h_{k}^{s}\right)^{*} h_{k}^{t}\right]=r^{|s-t|}$, and $0<r<1$ is the correlation between adjacent channel vectors. With the equal correlation model, all states have the same correlation with each other so that $\mathbf{E}\left[\left(h_{k}^{s}\right)^{*} h_{k}^{t}\right]=r$. Since the correlations with the exponential model decay as powers of $r$, there is less overall correlation between the states compared to the equal correlation model.

Consider the vector $\mathbf{h}=\left(h_{k}^{1}, h_{k}^{2}, \ldots, h_{k}^{S}\right)^{T}$ containing the k-th elements of $\mathbf{h}_{1}, \mathbf{h}_{2}, \ldots, \mathbf{h}_{S}$. The correlation matrix, $\mathbf{R}$, of $\mathbf{h}$ is defined by $(\mathbf{R})_{i j}=r^{|i-j|}$ for the exponential model and by $(\mathbf{R})_{i j}=r$ for the equal correlation model. When $r=0, \mathbf{h}$ contains $S$ independent channel elements and we have the gains due to reconfiguration discussed in Sections II and III.

When $r \neq 0$, the correlation between states will reduce the gains, but a direct analytical evaluation of the performance appears to be intractable. Consider the analysis in Section III-B where the maximum channel power is considered. In the correlated scenario, the mathematical framework for such a performance analysis is that of order statistics for identical but correlated variables. A description of the approach is given in [27, p. 99]. In addition to being cumbersome and involving multiple summations, the approach relies on the joint density of $S$ correlated Chisquared variables. Such a density involves infinite series and special functions even for $S=3$ 
[25] and is unknown for $S>4$. For this reason we do not pursue this direction further. However, a convenient way to quantify the effects of correlation can be found in [26]. Here, a metric was proposed to measure the number of independent channels (NIC) in a correlated channel vector, h. The metric can be defined as

$$
\mathrm{NIC}=\sum_{i=1}^{S} A_{i i}^{2},
$$

where the matrix $\mathbf{A}=A_{i j}$ is found from the Cholesky decomposition of the correlation matrix, $\mathbf{R}=\mathbf{A} \mathbf{A}^{\dagger}$. Using this metric, the performance of the reconfigurable system with $S$ correlated states can be approximated by that of a reconfigurable system with $S-K$ independent states where

$$
\sum_{i=1}^{S} A_{i i}^{2}=S-K
$$

For the exponential model, it is known that $\sum_{i=1}^{S} A_{i i}^{2} \geq S-(S-1) r^{2}$ [26] is a tight lower bound. This gives the particularly simple approximation that correlation results in a loss of $K$ independent states when $(S-1) r^{2}=K$. Hence, the correlation parameter

$$
r=\sqrt{\frac{K}{S-1}}
$$

results in an approximate loss of $K$ independent states. Although this result was derived from the exponential model, it is also useful for the equal correlation model as shown in Section V, where the accuracy of this approach is demonstrated.

\section{Simulation Results}

We use RVQ codebooks to compare the simulated and analytical results. Grassmannian codebooks [23] are used to explore the codebook expansion effect due to their availability in different sizes. Standard LTE codebooks are then used to demonstrate the improvements offered by reconfigurable antennas over non-reconfigurable systems.

Fig. 1 presents the channel direction and channel power results derived in the previous section for a $2 \times 1$ MISO system using a reconfigurable antenna with $S=2,8$, and RVQ codebooks. Note that the analytical results in (18) and (27) are given by the lines and simulations are plotted using points. It can be seen that they follow each other almost exactly. Also, we see that the 


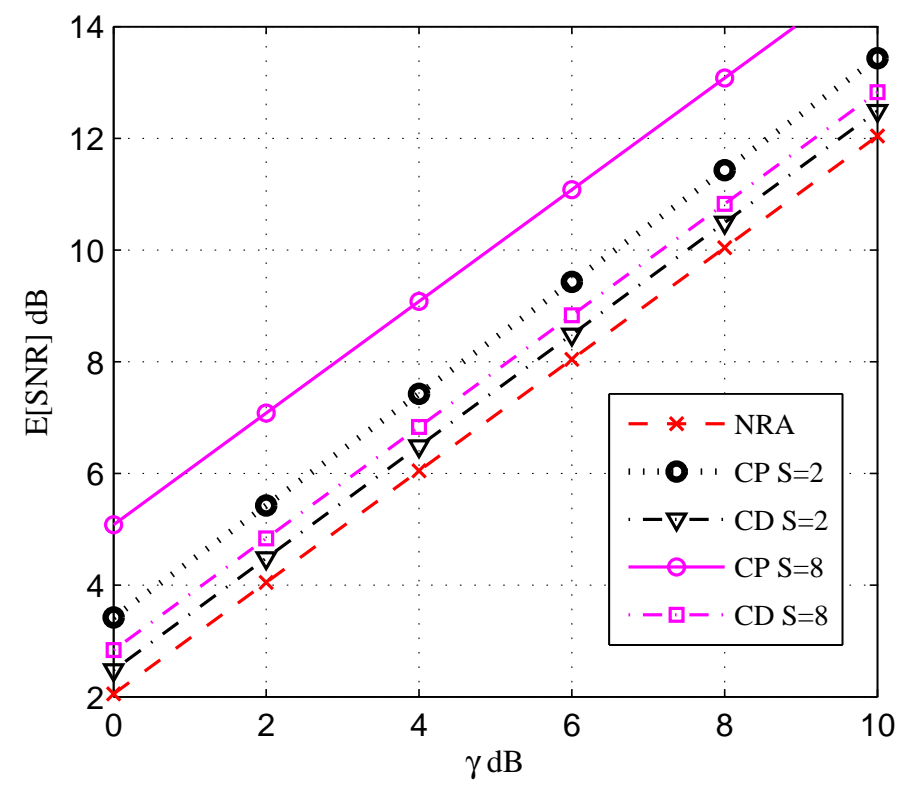

Fig. 1. Analytical (lines) and simulated (markers) results of E[SNR] for a $2 \times 1$ MISO reconfigurable codebook feedback system using RVQ codebooks with $S=2,8$ and using channel power (CP) and channel direction (CD) selection methods.

average SNR using channel power always significantly outperforms the use of channel direction and non-reconfigurable antennas (NRAs) with codebook feedback.

The SNR improvements due to reconfiguration are summarized in Table I. The percentage improvement values are calculated as $\left(\mathbf{E}\left[S N R_{S=2,4,8}\right]-\mathbf{E}\left[S N R_{S=1}\right]\right) / \mathbf{E}\left[S N R_{S=1}\right]$. Note that the $\%$ gains going from $S=1$ to $S=2$ for the channel power and optimum schemes are approximately half the gains observed in going from $S=1$ to $S=4$ and one third of the gains in going from $S=1$ to $S=8$. Hence, diminishing gains are occurring and doubling the number of states is required to maintain the same level of improvement. Also, it is apparent that the optimum approach gives the highest SNR and we can say that doubling $S$ gives approximately $40 \%$ gain.

Fig. 2 presents the SNR CDFs for $4 \times 1$ MISO systems with $2^{B}=16, S=16$ (dotted lines) and $2^{B}=64, S=4$ (solid lines). It clearly shows that the optimum approach has the best performance compared to the use of channel direction and channel power and that the channel direction method improves over a non-reconfigurable system with codebook feedback, but never exceeds perfect feedback unlike the channel power and optimum approaches Also, the gains of the optimum scheme over channel power are marginal. Note here that the expanded codebook 
TABLE I

AVERAGE SNR \% IMPROVEMENT OVER NON-RECONFIGURABLE ANTENNAS, USING DIFFERENT SELECTION METHODS IN A RECONFIGURABLE 1 X2 MISO SYSTEM WITH AN LTE CODEBOOK.

\begin{tabular}{c|c|c|c}
$S$ & $\begin{array}{c}\mathbf{E}[\mathrm{SNR}] \mathrm{CD} \\
\text { \%gain }\end{array}$ & $\begin{array}{c}\mathbf{E}[\mathrm{SNR}] \mathrm{CP} \\
\% \text { gain }\end{array}$ & $\begin{array}{c}\mathbf{E}[\mathrm{SNR}] \mathrm{OP} \\
\% \text { gain }\end{array}$ \\
\hline 1 & 0 & 0 & 0 \\
2 & 7 & 38 & 39 \\
4 & 11 & 77.5 & 79 \\
8 & 14 & 118 & 121
\end{tabular}

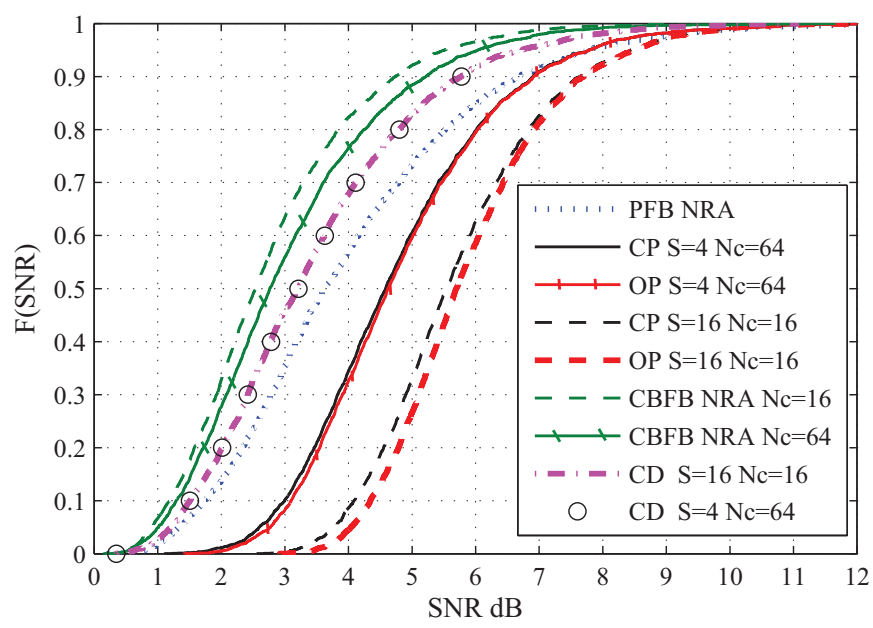

Fig. 2. SNR CDFs for $4 \times 1$ MISO reconfigurable codebook feedback system using Grassmannian codebooks [23] with $2^{B}=64, S=4$ (solid lines) and $2^{B}=16, S=16$ (dotted lines).

size is $2^{B} S=256$ for both cases, and as shown by the superimposed curves for channel direction in Fig. 2 and in (16), the $\mathrm{SNR}_{C D} \mathrm{CDFs}$ are equal. However, there is a higher selection gain for the optimum and channel power methods for $S=16$ while using a smaller codebook (4 bits), compared to $S=4$ and a 4 times larger codebook (6 bits).

Fig. 3 shows the QPSK symbol error rate (SER) comparison for non-reconfigurable (NRA, blue line) and reconfigurable systems with $S=2$ (RA, red lines) and $S=4$ (black lines) for codebook feedback MISO systems using optimum selection. First we consider the perfect CSI case (solid lines). For the $2 \times 1$ system at a SER of $10^{-2}$, the gain in $\gamma$ for $S=2$ is about $4 \mathrm{~dB}$ over non-reconfigurable codebook feedback and is $2 \mathrm{~dB}$ more for $S=4$. For the $4 \times 1$ system at a SER of $10^{-3}$, the gain in $\gamma$ for $S=2$ is about $3 \mathrm{~dB}$ over non-reconfigurable codebook feedback. It is about $1.7 \mathrm{~dB}$ for $S=4$ over $S=2$. We see that the SER gains diminish with 


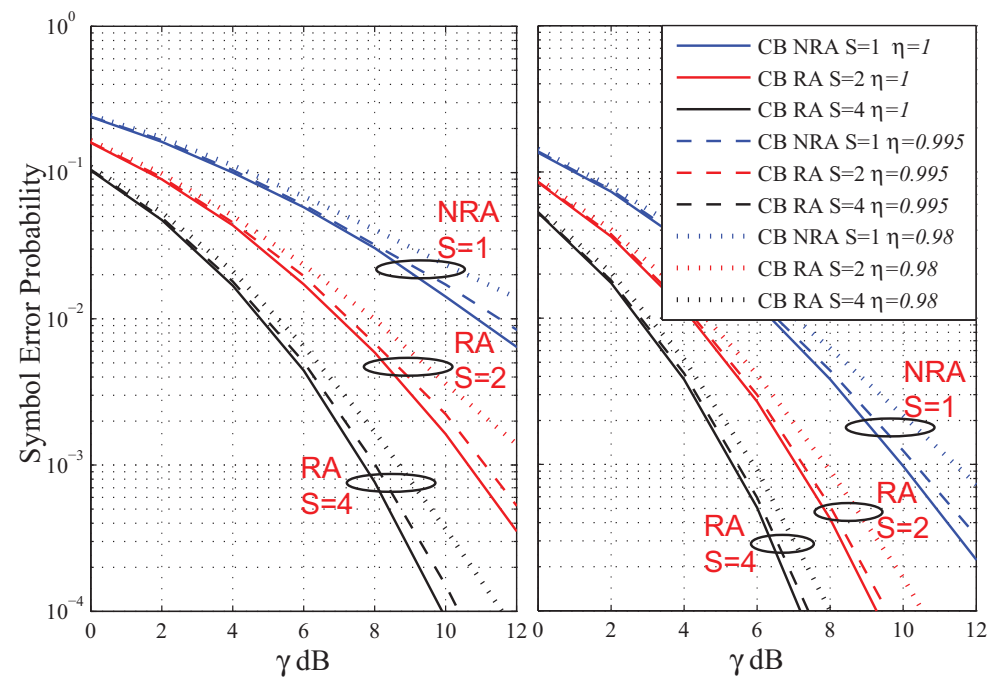

Fig. 3. SER performance results of $2 \times 1$ (left) and $4 \times 1$ (right) MISO systems using LTE codebooks with $2^{B}=4$ and $2^{B}=16, S=1,2,4$ and perfect and imperfect CSI.

increasing $S$. Also, comparing a smaller $2 \times 1$ system with $2^{B}=4$ and a larger $4 \times 1$ system, with $2^{B}=16$, we notice that the smaller system with $S=4$ and the larger system with $S=2$ have similar SER slopes at high $\gamma$, suggesting similar diversity. The curves with imperfect CSI in Fig. 3, for $\eta=0.995$ (dashed lines) and $\eta=0.98$ (dotted lines), show that reconfigurable antennas can still provide diversity gains with imperfect CSI.

Fig. 4 shows the rate CDFs for the MISO systems using optimum selection for $S=2,4,8$ and LTE codebooks. We see that for the $N_{t}=2$ system (left) with just $S=2$, the rate performance achieved, not only overcomes the quantization/codebook loss, but is always better than the rate achieved from the perfect feedback non-reconfigurable case. As $S$ increases, this gain over the perfect feedback non-reconfigurable case increases. However, in a $N_{t}=4$ system (right), reconfiguration with $S=2$ achieves a better rate than a perfect feedback non-reconfigurable antenna for $13 \%$ of the curve, $S=4$ for $70 \%$ of the curve and $S=8$ for $93 \%$ of the curve.

Figs. 5 and 6 show the impact of correlation between states on performance. In Fig. 5 we have a $4 \times 1$ system with $S \in\{1,2,3,4\}, \gamma=0 \mathrm{~dB}$ and $2^{B}=16$. The CDF of the SNR (labelled IID) is shown for independent states with $S \in\{1,2,3,4\}$. Clearly, the incremental performance losses increase as $S$ drops from 4 to 1 . Also shown are the CDFs of the SNR for $S=4$ with correlated states. Results for both the exponential (EXC) and equal (EQC) correlation models are shown 


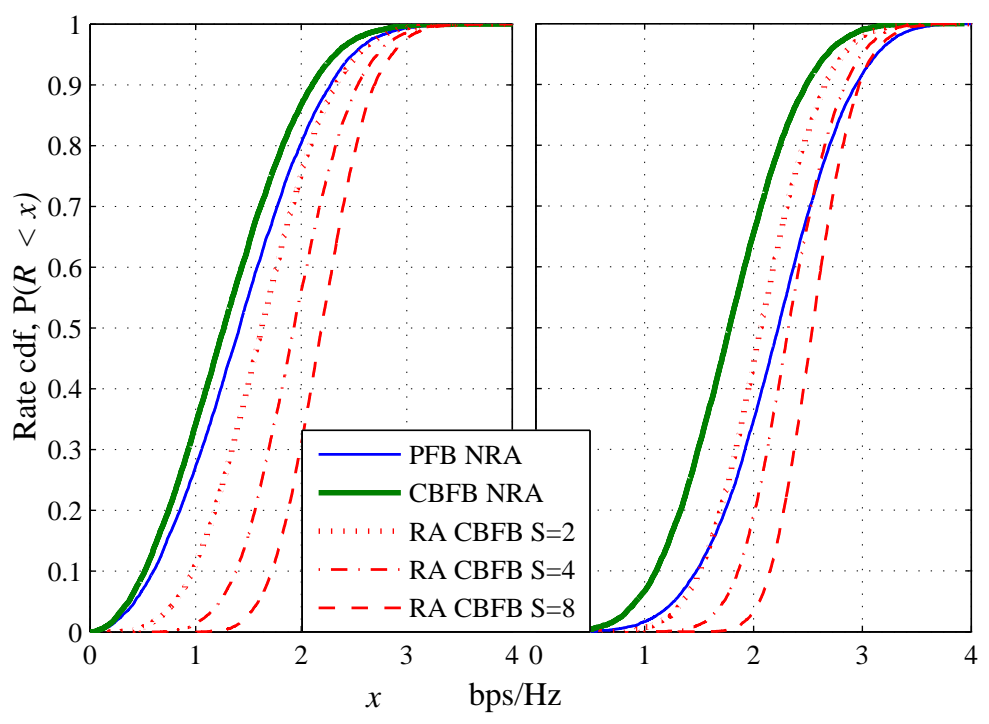

Fig. 4. Rate $\mathcal{R}$ CDFs for $2 \times 1$ (left) and $4 \times 1$ (right) MISO systems using optimum selection, with $S=2,4,8$ and LTE codebooks.

for $r=\sqrt{1 / 3}$ (corresponding to a loss of 1 state from (28)) and $r=\sqrt{2 / 3}$ (corresponding to a loss of 2 states). As expected, the equal correlation model gives worse performance than the exponential model, but the difference is not major. We note that the remarkably simple result in (28) gives a good indicator of performance as the CDFs for the correlated cases with $r=\sqrt{1 / 3}$ and $r=\sqrt{2 / 3}$ being similar to the CDFs for $S=3$ and $S=2$, respectively, for both exponential and equal correlation models. Note that when $K=S-1$, only one state remains and here, $r=1$, so we have exactly one independent state. Hence, the use of (28) is exact at the extremes of $K=0(r=0)$ and $K=S-1(r=1)$.

Fig. 6 shows a similar set of results for a $4 \times 1$ system with $S \in\{1,2,5,8\}, \gamma=0 \mathrm{~dB}$ and $2^{B}=8$. The same trends are observed as in Fig. 5 although the larger number of states $(S=8)$ spreads out the CDFs more than in Fig. 5. The CDFs for the correlated cases with $r=\sqrt{3 / 7}$ and $r=\sqrt{6 / 7}$, which model the loss of 3 and 6 states, are similar to the $S=5$ and $S=2$ i.i.d. cases. Hence, as before, using (28) gives a good indicator of performance, especially for the equal correlation model. 


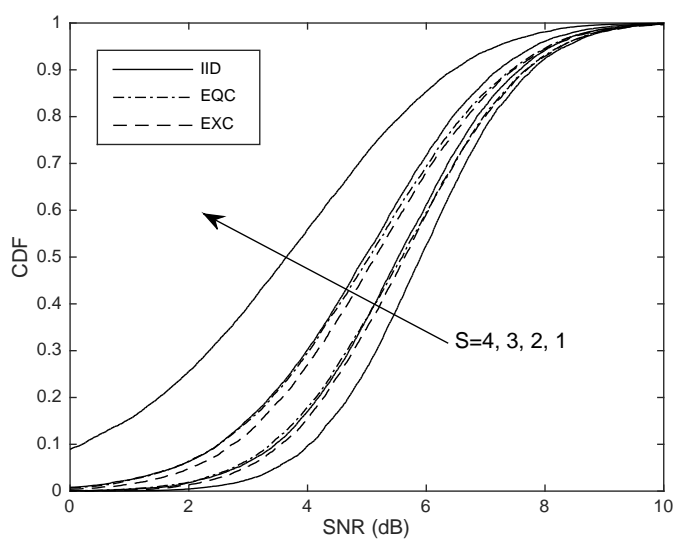

Fig. 5. SNR CDFs for i.i.d. states with $\mathrm{S}=1,2,3,4$ states. Also shown are the SNR CDFs for 4 exponential (EXC) or equally (EQC) correlated states with $r=\sqrt{1 / 3}$ and $r=\sqrt{2 / 3}$.

\section{CONCLUSION}

The reconfigurable codebook feedback system proposed, significantly improves the SNR, SER and the rate of the codebook feedback MISO system. Our analytical (16) and simulation results show that the CDF from using channel direction selection in a codebook feedback MISO system equipped with an $S$ state reconfigurable antenna at the receiver, is equivalent to that of an expanded codebook with size $2^{B} S$. Thus, a smaller codebook can be used to achieve the same 


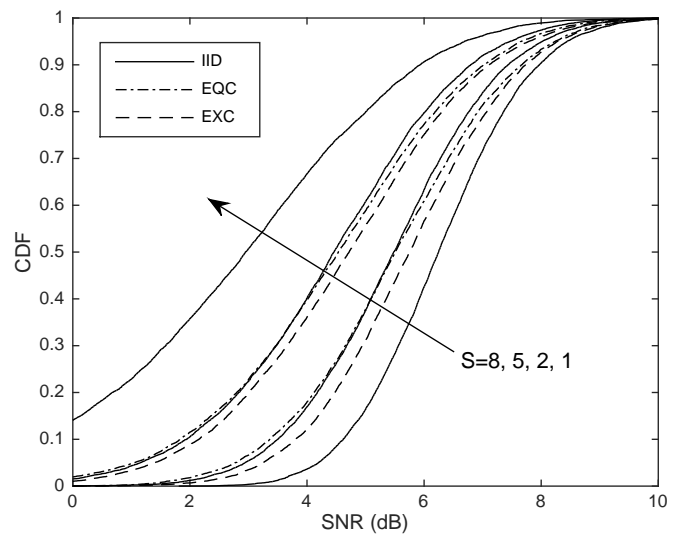

Fig. 6. SNR CDFs for i.i.d. states with $\mathrm{S}=1,2,5,8$ states. Also shown are the SNR CDFs for 8 exponential (EXC) or equally (EQC) correlated states with $r=\sqrt{3 / 7}$ and $r=\sqrt{6 / 7}$.

SNR performance as with a larger codebook and hence reduce the number of feedback bits.

In this paper, we consider three reconfigurable antenna codebook selection techniques and have demonstrated that the channel direction approach is considerably worse than the channel power scheme which is close to optimum in terms of both SNR and rate performance. The expected SNR expressions for the channel direction and channel power schemes are also given from which we conclude that the traditional channel direction approach provides only a very 
small part of the gains offered by the optimum scheme. When using a system with reconfigurable antenna, codebook feedback and optimum selection, we also show that as the number of states increase, the gain in SNR, SER and the rate of the system increases. Their CDFs show that they not only overcome the codebook loss, but the gain exceeds that achieved by a non-reconfigurable antenna with perfect feedback. SER curves show that the SER slope of the system improves as $S$ increases. Also a larger $4 \times 1$ system with $2^{B}=16, S=2$ and a smaller $2 \times 1$ system with $2^{B}=4$ and $S=4$ have similar SER slopes at high $\gamma$. By doubling $S$ the gain in SNR is approximately $40 \%$ as compared to $S=1$. The overall SER conclusions remain the same for the imperfect CSI cases considered ( $\eta=0.995,0.98)$, which are more realistic scenarios and require less training overhead. Thus, using the proposed reconfigurable codebook feedback system with optimum selection, the codebook loss due to quantization can be significantly reduced with only $S=2$ and the resulting SNR and rate can exceed those of the perfect feedback traditional fixed antenna system. Finally, we show that a simple closed form result can be used to measure the number of equivalent independent states in the correlated case. The performance of a system with this reduced, equivalent, number of independent states is shown to be close to the performance of the full system with correlated states.

\section{REFERENCES}

[1] Anagnostou, D.E., Zheng, G., Chryssomallis, M.T., et. al.: 'Design, fabrication, and measurements of an RF-MEMS-based self-similar reconfigurable antenna', IEEE Transactions on Antennas and Propagation, 2006, 54 (2), pp. $422-432$

[2] Jung, C.W., Lee, M.-J., Li, G.P. and De Flaviis, F.: 'Monolithic integrated re-configurable antenna with RF-MEMS switches fabricated on printed circuit board', Proc. IECON, November 2005, pp. 1-6

[3] Grau, A., Romeu, J., Jofre, L. and De Flaviis, F.: 'On the polarization diversity gain using the ORIOL antenna in fading environments', Proc. AP-S Intl. Symp., July 2005, pp. 14-17

[4] Sayeed, A., Raghavan, V. and Kotecha, J.: 'Capacity of space-time wireless channels: A physical perspective', Proc. IEEE Inform. Theory Workshop, 2004, pp. 434-439

[5] Cetiner, B., Akay, E., Sengul, E. and Ayanoglu, E.: 'A MIMO system with multifunctional reconfigurable antennas', IEEE Antennas Wireless Propag. Lett., 2006, 5(1), pp. 463-466

[6] Migliore, M., Pinchera, D. and Schettino, F.: 'Improving channel capacity using adaptive MIMO antennas', IEEE Trans. Antennas Propag., 2006, 54(11), pp. 34813489

[7] Piazza, D., Kirsch, N., Forenza, A., Heath, R. and Dandekar, K.: 'Design and evaluation of a reconfigurable antenna array for MIMO systems', IEEE Trans. Antennas Propag., 2008, 56(3), pp. 869-881

[8] Mehmood, R. and Wallace, J.: 'Diminishing returns with increasing complexity in reconfigurable aperture antennas', IEEE Antennas Wireless Propag. Lett., 2010, 9, pp. 299-302 
[9] Love, D.J., Heath, R.W., Santipach, W. and Honig, M.L.: 'What is the value of limited feedback for MIMO channels?', IEEE Wireless Communication Magazine, 2004, 42 (10), pp.54-59

[10] Love, D.J., Heath, R.W., Lau, V.K.N., Gesbert, D., Rao, B.D. and Andrews, M.: 'An overview of limited feedback in wireless communication systems', IEEE Journal on Selected Areas of Communications, 2008, 26 (8), pp. 1341-1365

[11] 3GPP TS 36.211, 'Evolved universal terrestrial radio access (E-UTRA), physical channels and modulation', vol. V10.0.0, January 2011

[12] Love, D. J., Heath, R. W. and Strohmer, T.: 'Grassmannian beamforming for multiple-input multiple-output wireless systems', IEEE Trans. Inform. Theory, 2003, 49 (10), pp. 2735-2747

[13] Dabbagh, A. and Love, D.: 'Feedback rate-capacity loss tradeoff for limited feedback MIMO systems', IEEE Trans. Inform. Theory, 2006, 52(5), pp. 2190-2202

[14] Mondal, B. and Heath, R.: 'Performance analysis of quantized beamforming MIMO systems', IEEE Trans. Sig. Proc., 2006, 54(12), pp. 4753-4766

[15] Au Yeung, C. and Love, D.: 'On the performance of random vector quantization limited feedback beamforming in a MISO system', IEEE Trans. Wireless Commun., 2006, 6(1), pp, 458-462

[16] Zheng, J., Duni, E. and Rao, B.: 'Analysis of multiple antenna systems with finite rate feedback using high resolution quantization theory', IEEE Trans, Sig. Proc. 2007, 55(4), pp. 1461-1476

[17] Santipach, W. and Honig, M.: 'Capacity of multi-antenna fading channel with quantized precoding matrix', IEEE Trans. Inform. Theory, 2009, 55(3), pp. 1218-1234

[18] Zheng, J. and Rao, B.: 'Analysis of multiple antenna systems with finite-rate channel information feedback over spatially correlated fading channels', IEEE Trans. Sig. Proc., 2007, 55(9), pp. 4612-4626

[19] Raghavan, V. and Veeravalli, V.: 'Ensemble properties of RVQ-based limited-feedback beamforming codebooks', IEEE Trans. Inform. Theory, 2013, 59(12), pp. 8224-8249

[20] Mirza, J., Dmochowski, P.A., Smith, P.J. and Shafi, M.: 'Capacity loss for multilayer codebook precoding in MIMO systems', Proc. PIMRC, September 2012, pp. 1890-1895

[21] Yeung, C. K. A., Love, D.J.: 'On the performance of random vector quantization limited feedback beamforming in a MISO system', IEEE Trans. Wireless Commun., 2007, 6 (2), pp. 458-462

[22] Grant, S. J. and Cavers, J. K.: 'Multi-user channel estimation for detection of cochannel signals', IEEE Transactions on Communications, 2001, 49 (10), pp. 1845-1855

[23] Love D. J.: 'Grassmannian subspace packing', 2014, https://engineering.purdue.edu/ djlove/grass.html

[24] Loyka, S.L.: 'Channel capacity of MIMO architecture using the exponential correlation matrix', IEEE Communications Letters, 2001, 5(9), pp. 369-371

[25] Hagedorn, M., Smith, P. J., Bones, P. J., Millane, R. P. and Pairman, D.: 'A trivariate chi-squared distribution derived from the complex Wishart distribution', Journal of Multivariate Analysis, 2006, 97(3), pp. 655-674

[26] Smith, P.J., Dmochowski, P.A., Chiani, M. and Giorgetti, A.: 'On the number of independent channels in multi-antenna systems', IEEE Trans. Wireless Commun., 2014, 13(1), pp. 75-85

[27] David, H.A. and Nagaraja, H.N.: Order Statistics, Third Edition, John Wiley \& Sons, Inc, Hoboken, New Jersey, 2003 